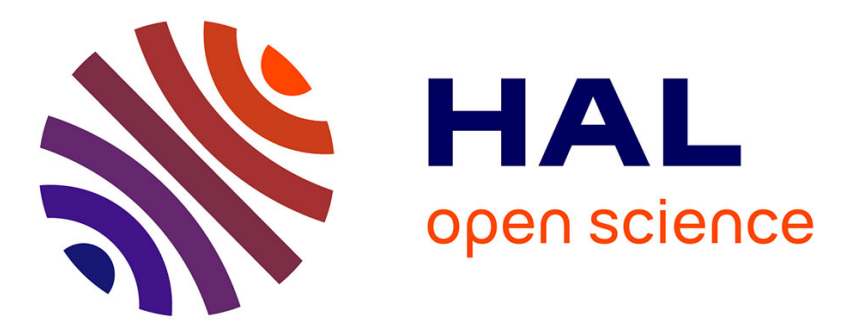

\title{
Identification of ion currents components generating field potential recorded in MEA from hiPSC-CM
}

Fabien Raphel, Muriel Boulakia, Nejib Zemzemi, Yves Coudière, Jean-Michel Guillon, Philippe Zitoun, Jean-Frédéric Gerbeau

\section{- To cite this version:}

Fabien Raphel, Muriel Boulakia, Nejib Zemzemi, Yves Coudière, Jean-Michel Guillon, et al.. Identification of ion currents components generating field potential recorded in MEA from hiPSC-CM. IEEE Transactions on Biomedical Engineering, 2018, 65 (6), pp.1311-1319. 10.1109/TBME.2017.2748798 . hal-01570341

\section{HAL Id: hal-01570341 https://hal.science/hal-01570341}

Submitted on 29 Jul 2017

HAL is a multi-disciplinary open access archive for the deposit and dissemination of scientific research documents, whether they are published or not. The documents may come from teaching and research institutions in France or abroad, or from public or private research centers.
L'archive ouverte pluridisciplinaire HAL, est destinée au dépôt et à la diffusion de documents scientifiques de niveau recherche, publiés ou non, émanant des établissements d'enseignement et de recherche français ou étrangers, des laboratoires publics ou privés. 


\title{
Identification of ion currents components generating field potential recorded in MEA from hiPSC-CM
}

\author{
Fabien Raphel, Muriel Boulakia, Nejib Zemzemi, Yves Coudière, \\ Jean-Michel Guillon, Philippe Zitoun, Jean-Frédéric Gerbeau*
}

July 29, 2017

\begin{abstract}
Objective: Multi Electrodes Arrays (MEAs) combined with cardiomyocytes derived from human induced pluripotent stem cells (hiPSC-CMs) can enable high- or medium-throughput drug screening in safety pharmacology. This technology has recently attracted a lot of attention, in particular from an international initiative named CiPA. But it is currently limited by the difficulty to analyze the measured signals. We propose a strategy to analyze the signals acquired by the MEA and to automatically deduce the channels affected by the drug.

Methods: Our method is based on the bidomain equations, a model for the MEA electrodes, and an inverse problem strategy.

Results: In silico MEA signals are obtained for two commercial devices and an example of Early After Depolarization (EAD) is presented. Then, by processing real signals obtained for four different compounds, our algorithm was able to provide dose-response curves for potassium, sodium and calcium channels. For ivabradine and moxifloxacin, the IC50 and dose-response curves are in very good agreement with known values.

Significance: The proposed strategy offers a possible answer to a major question raised by the community of safety pharmacology. By allowing a more automated analysis of the signals, our approach could contribute to promote the technology based on MEA and hiPSC-CMs, and therefore improve reliability and efficiency of drug screening.
\end{abstract}

\section{Introduction}

The objectives of Safety Pharmacology studies are to characterize, at the preclinical stage, the potential pharmacodynamic effects of drug candidates on the

${ }^{*}$ F. Raphel, M. Boulakia and J-F. Gerbeau, Inria Paris and Sorbonne Universités UPMC, Paris, France; N. Zemzemi and Y. Coudière, Inria Bordeaux and Université Bordeaux 1, France; P. Zitoun, LIPZ, La Celle Saint Cloud, France; J-M. Guillon, sanofi R\&D, preclinical safety, Vitry sur Seine, France 
main physiological functions, in the therapeutic range and above. This preclinical discipline is mainly focused on the studies of unwanted effects of drug candidates on cardiovascular and central nervous systems. The methods used to achieve these missions cover a wide spectrum, spanning from in vitro to in vivo models as well as to in silico modeling as tools used to anticipate the potential issues as early as possible in development process. Amongst the cardiovascular side effects that are studied, the electrophysiological effects of drug candidates are of major importance since a significant part of drug development attrition and drug market withdrawal are due to arrhythmogenic potential. An international initiative under the umbrella of the US Food and Drug Administration, Cardiac Research Safety Consortium, ILSI Health and Environmental Sciences Institute, and Safety Pharmacology Society is aimed to address the propensity for a drug to be pro-arrhythmic and/or arrhythmogenic. This initiative was named $\mathrm{CiPA}$ for Comprehensive in vitro Pro-Arrhythmias assay $[5,6]$. The CiPA paradigm has been designed to provide an accurate and comprehensive assessment of the cardiac ventricular electrophysiological properties of drugs candidates for identifying mechanisms that may mediate life-threatening ventricular arrhythmias. The CiPA core components are the following: (i) In vitro patch clamp assays using stably expressed recombinant human ion channels for evaluating the effects of candidate drugs on major ion currents contributing to human ventricle action potential (AP). (ii) An in silico AP assay is performed to verify whether the integration of the data obtained in the patch-clamp assays is indicative of pro-arrhythmic events on the human ventricular AP reconstructed according to the O'Hara-Rudy model. (iii) An in vitro assay investigating the effects of drug candidates on electrophysiological endpoints in ventricular cardiomyocytes derived from human induced pluripotent stem cells (hiPSC-CMs). A goal of this assay is to confirm and complement (if necessary) the in silico predictions. One of the models selected for this purpose is the recording and analysis of the electrical potential recorded from spontaneously beating hiPSCCMs maintained in culture in Multi Electrodes Arrays (MEA).

But several challenges remain. It is still difficult to obtain hiPSC-CM populations which are homogeneous and clearly differentiated into ventricular or atrial cardiac cells with a known amount of fibroblasts. In addition, the electrical potential measured with the MEA, called Field Potential (FP), is extracellular and is therefore less familiar and much more difficult to analyse than the AP.

The purpose of this article is to show that mathematical modeling and computer simulations may help decipher the FP and therefore contributes to promote this promising technology. By proposing an in silico approach of FP, this work can be viewed as complementary of the current approaches of CiPA. It is a step toward the automated identification of channel activity inferred from FP signals. Combined with the high-throughput capabilities of the MEAs, it offers a new strategy to evaluate the proarrhythmic risk of a drug.

Many in silico models have been proposed in the literature for the AP, based on patch-clamp measurements (e.g. $[17,18,20])$, and several experimental studies of the FP of hiPSC-CMs have been recently published (e.g. [3,14]). But to our knowledge, in silico models of FP are still missing. We recently proposed such 
a model [1], where the effect of drugs in a heterogeneous media was investigated through computer simulations. The present study is based on the same model, but its main focus is to tackle the inverse problem of identifying some parameters of the ionic currents from FP measurements.

The paper is organized as follows. The mathematical model, the MEA geometries and the parameter identification strategy are presented in Section 2. Section 3 first shows some simulations of the forward model. Contrary to [1], it is assumed here that the cells are homogeneous. This allows us to focus on other sources of variability in the signals like the spatial propagation and the device geometry. Two MEA devices are considered and the parameters of the electrode model are discussed. Then a verification of the inverse problem is performed on synthetic data and a discussion about the interest of using the measurements given by several electrodes is proposed. Finally, the algorithm is tested on four experimental datasets involving moxifloxacin, ivabradine, SEA0400 and diltiazem.

\section{Methods}

The mathematical model used in this work has been recently introduced in [1]. In this section, we briefly present the model and the geometrical setting of two commercial MEAs. Then, an inverse problem strategy is presented to identify the parameters of the in silico model from FP signals.

\subsection{Modeling of the cardiac electrical activity in the MEA}

We make the hypothesis that the cardiomyocytes are homogeneous and spread uniformly on a thin layer. The domain $\Omega$ representing the MEA and the cells is therefore assumed to be two-dimensional. Our model is composed of three components: a description of the ionic activity at the cell scale, a description of the spatial electrical propagation at the MEA scale, and a model of the microelectrodes.

\subsubsection{The ionic model}

There is a large variety of models of the transmembrane ionic current $I_{\text {ion }}$. They greatly differ in their level of details [7]. In the present work, three different ionic models have been considered: Minimal Ventricular (MV) [4], Paci et al. [18] and O'Hara et al. [17]. The reader is referred to the original publications for the details. Schematically, the ionic current $I_{\text {ion }}$ depends on the transmembrane potential $V_{\mathrm{m}}$ and on ionic variables $w=\left(w_{j}\right)_{1 \leq j \leq M}$, solution of a system of nonlinear ordinary differential equations. It is given by $I_{\text {ion }}=\sum_{S} I_{S}$ where $I_{S}$, the current carried by an ionic species $S$, has the general expression

$$
I_{S}=g_{S} \prod_{j=1}^{M} w_{j}^{p_{j}}\left(V_{\mathrm{m}}-V_{\mathrm{m} S}\right) .
$$


Here, $g_{S}$ is a conductance coefficient, $V_{\mathrm{m} S}$ is the Nernst equilibrium potential of species $S$ and $p_{j}$ are integers.

The MV model [4] describes a ventricular human cell in a simplified manner through only three currents: fast inward, slow inward and outward currents. It is based on the electrophysiological analysis of an AP that is the end-results of several inward and outward currents carried by several ionic species flowing through specific ion channels (the gating of which being mainly controlled by membrane potential). The Paci model [18] describes a hiPSC-CM and the O'Hara-Rudy model [17] describes a human ventricular cells derived from a large set of experiments with human cardiomyocytes. The advantage of physiological models, like these two, is that they give a detailed description of several ionic currents. An interesting capability of the O'Hara-Rudy model is its capability to model EADs, that can be viewed as an electrical signature of arrhythmias occurrence. However, their parameters can be difficult to identify. For inverse problems, MV therefore offers an interesting alternative.

The Paci model was slightly modified because the spontaneous beating of this model leads to an homogeneous depolarization in the whole domain which prevents the propagation of the signal: the current $I_{f}$ was set to zero and the sodium current was replaced by the O'Hara-Rudy one.

\subsubsection{The bidomain model}

To represent the spatial propagation of the potentials in $\Omega$, we consider the bidomain model [21]. This model describes the evolution of the transmembrane potential $V_{\mathrm{m}}$ and the extracellular potential $\phi_{\mathrm{e}}$ through two coupled partial differential equations which can be found in the Supplementary Material S1.

\subsubsection{Modeling of the microelectrodes}

The field potential measured by an electrode $e_{k}$ is given by $\phi_{\mathrm{f}}^{k}=R_{\mathrm{i}} I_{\mathrm{el}}^{k}$ where $R_{\mathrm{i}}$ is the internal resistance of the measurement device. Denoting by $C_{\mathrm{el}}$ and $R_{\mathrm{el}}$ the electrode capacitance and the electrode resistance, current $I_{\mathrm{el}}^{k}$ is linked to the mean extracellular potential $\phi_{\mathrm{e}, \text { mean }}^{k}$ at the electrode by the equation

$$
\frac{d I_{\mathrm{el}}^{k}}{d t}+\frac{1}{\tau} I_{\mathrm{el}}^{k}=\frac{C_{\mathrm{el}}}{\tau} \frac{d}{d t} \phi_{\mathrm{e}, \text { mean }}^{k}
$$

where $\tau=\left(R_{\mathrm{el}}+R_{\mathrm{i}}\right) C_{\mathrm{el}}$.

\subsubsection{Geometrical modeling of two commercial devices}

Two kinds of devices are considered: a 96-well MEA by Axion BioSystems ${ }^{\mathrm{TM}}$ and a 6 -well MEA by Multichannel Systems ${ }^{\mathrm{TM}}$. Since the wells are independent from each other, the simulations are run on a single well. In the 96-well MEA, the disc-shaped well is made of 8 microelectrodes and 4 grounds around the electrodes. In the 6-well MEA, the square-shaped well is made of 9 microelectrodes and a ground at three sides of the square. The finite element meshes used in the numerical simulation are reported in the Supplementary Material S2. 


\subsection{Identification of ionic currents from FPs}

To identify the channel conductances of the ionic model from FP measurements, we minimize a cost function which quantifies the discrepancy between the real and the in silico field potentials. To do so, two types of cost functions have been considered: one with the whole FP signals on a given time interval, the other with only a few metrics (biomarkers) extracted from the signals.

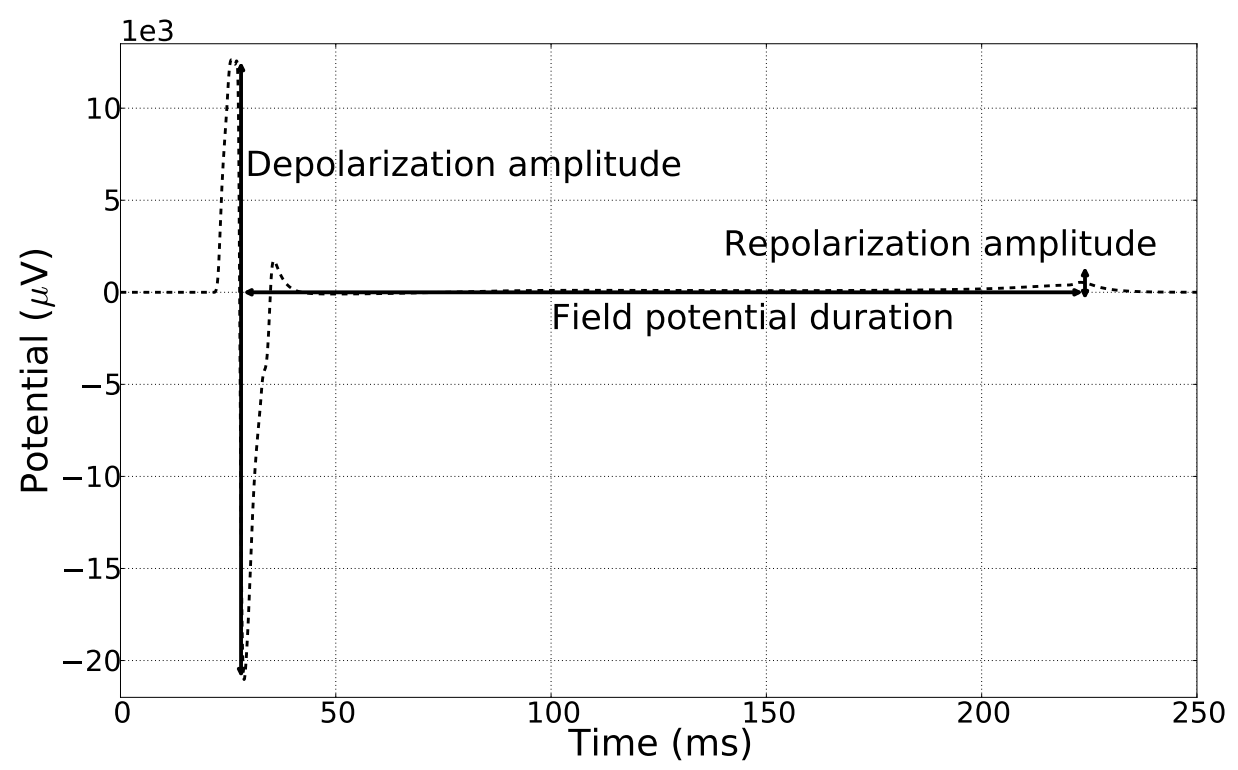

Figure 1: Biomarkers used in the inverse problems: depolarization amplitude (DA), repolarization amplitude (RA) and field potential duration (FPD)

Let us present the two cost functions more precisely. Assume that the measurements of the FPs are available at $N$ electrodes during a time interval $[0, T]$. The purpose is to identify some ionic model parameters, denoted by $\theta$. In the examples presented below, $\theta$ is a vector of channel conductances $\left(g_{N a}, g_{K}, g_{C a}\right)$ appearing in the definition (1) of the ionic currents $I_{N a} I_{K}$ and $I_{C a}$.

For the first cost function, we denote by $\phi_{\mathrm{f}, \text { ref }}^{k}$ the reference FP measured at the $k$-th electrode which is a time-dependent function and we minimize the following quantity with respect to $\theta$ :

$$
J_{1}(\theta)=\sum_{k=1}^{N} \int_{0}^{T}\left|\phi_{\mathrm{f}, \text { ref }}^{k}(t)-\phi_{\mathrm{f}}^{k}(\theta, t)\right|^{2} d t,
$$

where $\phi_{\mathrm{f}}^{k}(\theta, \cdot)$ is the time-dependent field potential $\phi_{\mathrm{f}}$ taken at the $k$-th electrode given by the resolution of the bidomain equations, (2) and the ionic model with the parameter value $\theta$. 
For the second cost function, we introduce three biomarkers represented in Fig. 1: depolarization amplitude (DA), repolarization amplitude (RA) and field potential duration (FPD). In the cost function to optimize, the values of these biomarkers are rescaled with the biomarkers of a control case. This renormalization is important in practice: it makes less critical to precisely fit the amplitude of the experimental signal. The biomarkers associated with the reference signal $\phi_{\mathrm{f}, \text { ref }}^{k}$ are denoted by $\mathrm{DA}_{\text {ref }}^{k}, \mathrm{RA}_{\text {ref }}^{k}$ and $\mathrm{FPD}_{\text {ref }}^{k}$ and the biomarkers associated with the reference control signal by $\mathrm{DA}_{\mathrm{c}, \mathrm{ref}}^{k}$, $\mathrm{RA}_{\mathrm{c} \text {,ref }}^{k}$ and $\mathrm{FPD}_{\mathrm{c}, \mathrm{ref}}^{k}$. Then, the following quantity is minimized with respect to $\theta$ :

$$
\begin{aligned}
& J_{2}(\theta)=\sum_{k=1}^{N}\left(\frac{\mathrm{DA}_{\mathrm{ref}}^{k}}{\mathrm{DA}_{\mathrm{c}, \mathrm{ref}}^{k}}-\frac{\mathrm{DA}^{k}(\theta)}{\mathrm{DA}_{\mathrm{c}}^{k}}\right)^{2}+ \\
& \left(\frac{\mathrm{RA}_{\mathrm{ref}}^{k}}{\mathrm{RA}_{\mathrm{c}, \mathrm{ref}}^{k}}-\frac{\mathrm{RA}^{k}(\theta)}{\mathrm{RA}_{\mathrm{c}}^{k}}\right)^{2}+\left(\frac{\mathrm{FPD}_{\text {ref }}^{k}}{\mathrm{FPD}_{\mathrm{c}, \mathrm{ref}}^{k}}-\frac{\mathrm{FPD}^{k}(\theta)}{\mathrm{FPD}_{\mathrm{c}}^{k}}\right)^{2}
\end{aligned}
$$

where $\operatorname{DA}^{k}(\theta), \operatorname{RA}^{k}(\theta)$ and $\operatorname{FPD}^{k}(\theta)$ are the biomarkers of the signal $\phi_{\mathrm{f}}^{k}(\theta, \cdot)$ and $\mathrm{DA}_{\mathrm{c}}^{k}, \mathrm{RA}_{\mathrm{c}}^{k}$ and $\mathrm{FPD}_{\mathrm{c}}^{k}$ are the biomarkers of the control signal given by the model.

\section{$2.3 \quad$ Numerical methods}

Our in silico assays are based on the resolution of the bidomain equations coupled to (2) and to one of the ionic models presented in section 2.1.1. The space discretization is done with $\mathrm{P} 1$ finite element. The time discretization is done with a Strang splitting, and a second order Backward Differentiation Formula (BDF2), with variable time steps and inexact Newton method, provided by the Sundials library [12]. The optimization problems corresponding to the parameter identification are solved with the evolutionary algorithm CMA-ES [11].

\section{Results}

\subsection{Numerical field potentials}

In the following simulations, the parameters of the bidomain model are: $A_{\mathrm{m}}=$ $1400 \mathrm{~cm}^{-1}, C_{\mathrm{m}}=1 \mu \mathrm{F} \mathrm{cm}^{-2}, \sigma_{\mathrm{i}}=0.7 \mu \mathrm{S} \mathrm{cm}^{-2}, \sigma_{\mathrm{e}}=1.2 \mu \mathrm{S} \mathrm{cm}^{-2}$. And the electrode parameters are:

$$
R_{\mathrm{i}}=290 M \Omega, R_{\mathrm{el}}=40 M \Omega \text { and } C_{\mathrm{el}}=2 \mu F .
$$

In Figures 2 and 3, we have represented the numerical field potentials and transmembrane potentials measured at several electrodes of a disc-shaped well with 8 microelectrodes (Figures 2) and a square-shaped well with 9 microelectrodes (Fig. 3). These simulations have been performed with the Paci model [18], slightly modified as explained above. We observe differences in the shape of FPs between the two MEAs: in the disc-shaped well, the amplitude of the signal is larger, less stiff and the repolarization phase is more visible than in the squareshaped. In Fig. 3, it is worth noticing that the FP shape varies in amplitude and orientation depending on the electrode. 

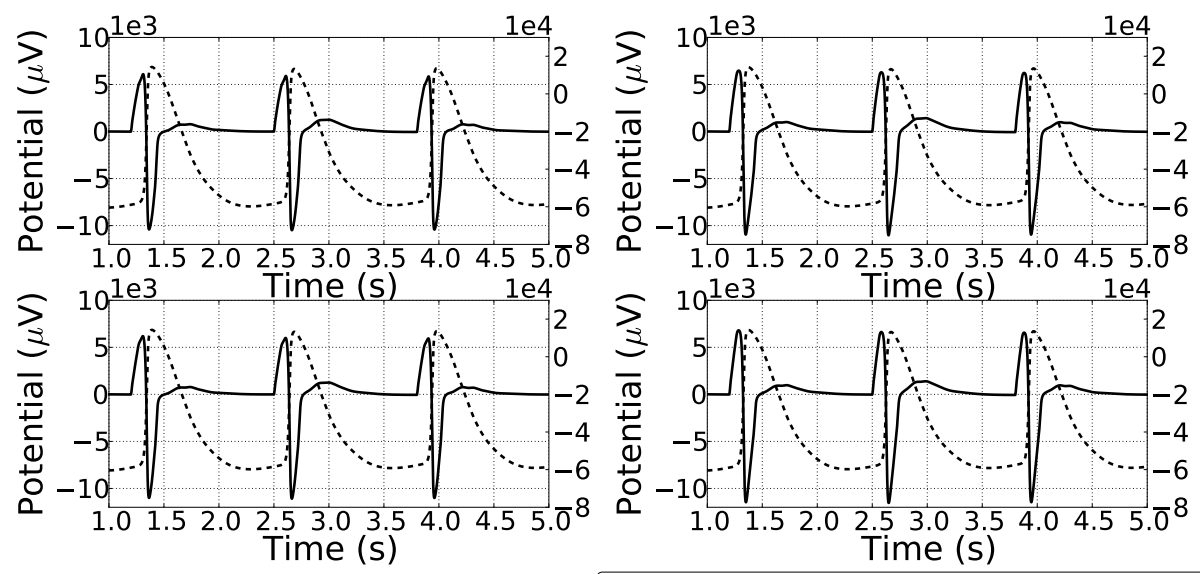

Field potential scale on the left axis, action potential scale on the right axis.

Figure 2: In silico transmembrane potential (dotted line) and field potential (continuous line) at 4 different electrodes in a disc-shaped well with 8 microelectrodes, obtained by periodic stimulations.
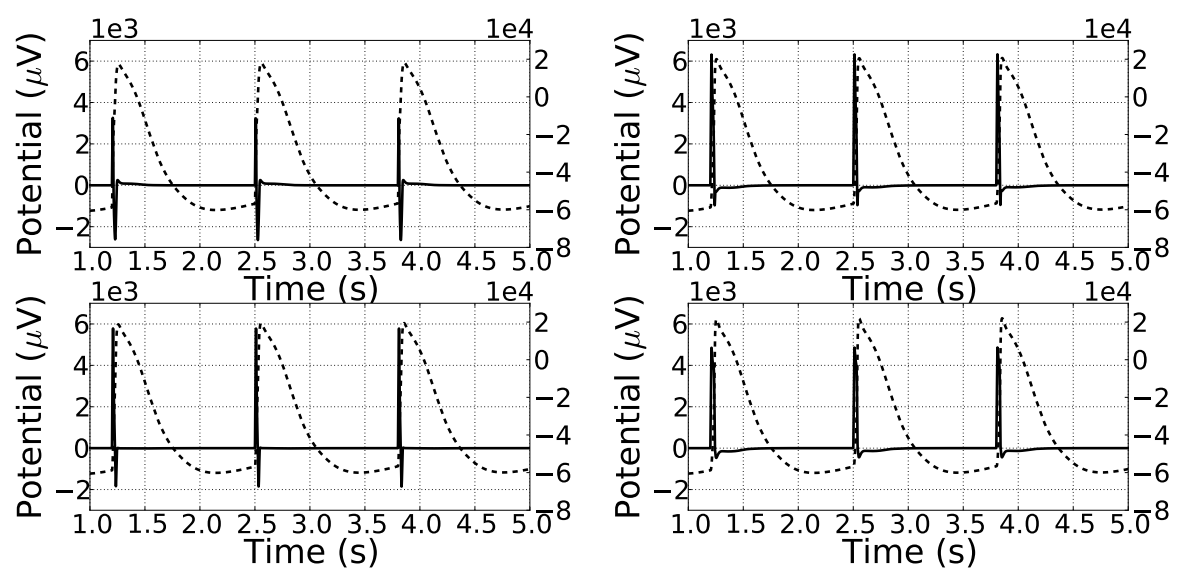

Field potential scale on the left axis, action potential scale on the right axis.

Figure 3: In silico transmembrane potential (dotted line) and field potential (continuous line) at 4 different electrodes in a square-shaped well with 9 microelectrodes, obtained by periodic stimulations. 
The FP spatial variability is even more apparent in Fig. 4. This figure illustrates the dependence of the signal measured at an electrode with respect to the distance between the electrode and the location of the initial stimulation, modeled by the applied current $I_{\mathrm{app}}$. It was also observed that the closer is the initial stimulation to the measurement electrode, the larger is the negative peak in depolarization. In [3], FPD recorded with a MED64 was found to be roughly equal to APD50 estimated with voltage-sensitive dye. As a first approximation, our simulations are not far from this observation, but the precise relationship seems to depend on the device, the electrode and the drug concentration (see Supplementary material S3).
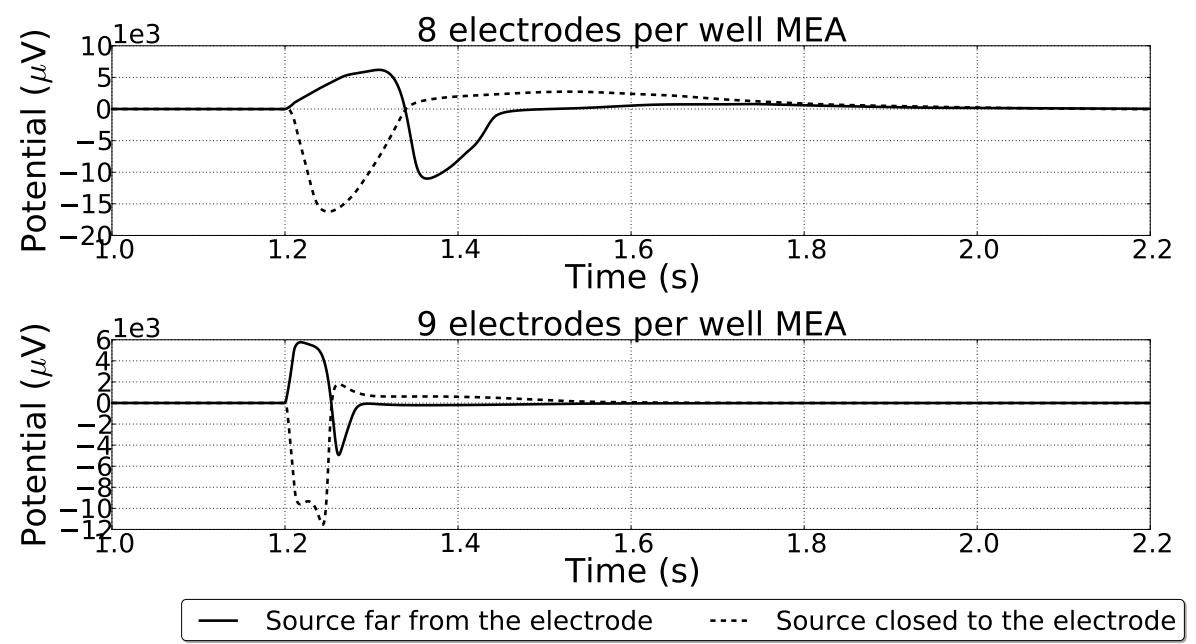

Figure 4: In silico field potentials for different source localizations

The influence of the electrode parameters is illustrated in Figures 5 and 6 where the depolarization phase of the field potential is plotted for different values of $R_{\mathrm{el}}$ and $C_{\mathrm{el}}$.

As indicated in Fig. 5, the increase of the resistance $R_{\mathrm{el}}$ induced a decrease in the signal amplitude. The same observation was made with the resistance $R_{\mathrm{i}}$. The decrease of the capacitance $C_{\mathrm{el}}$ induced a distortion of the signal and a decrease of the signal amplitude (Fig. 6). With the ranges of values considered for the resistance and capacitance (5), their effect is very limited and the field potential $\phi_{\mathrm{f}}$ is very similar to the extracellular potential $\phi_{\mathrm{e}}$. Varying the resistances or conductances in our model could be a way to model the quality of the adhesion of the cells to their support. This aspect was not further investigated in the present study. 


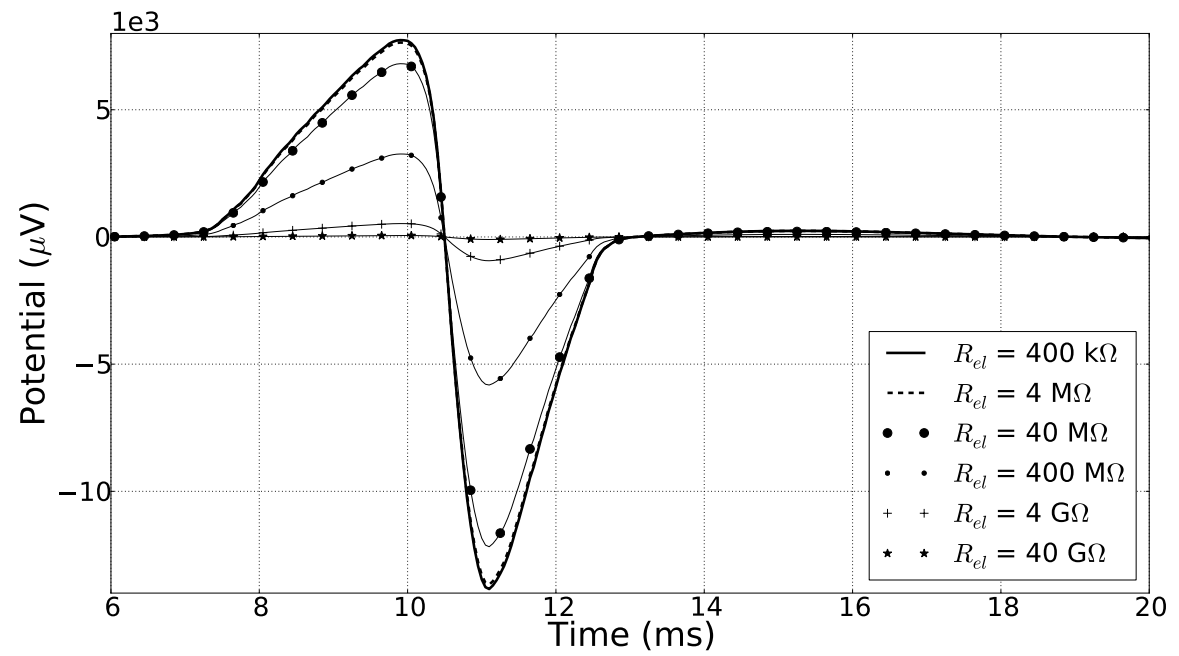

Figure 5: In silico depolarization phase of the field potential for different values of the electrode resistance $R_{\mathrm{el}}$.

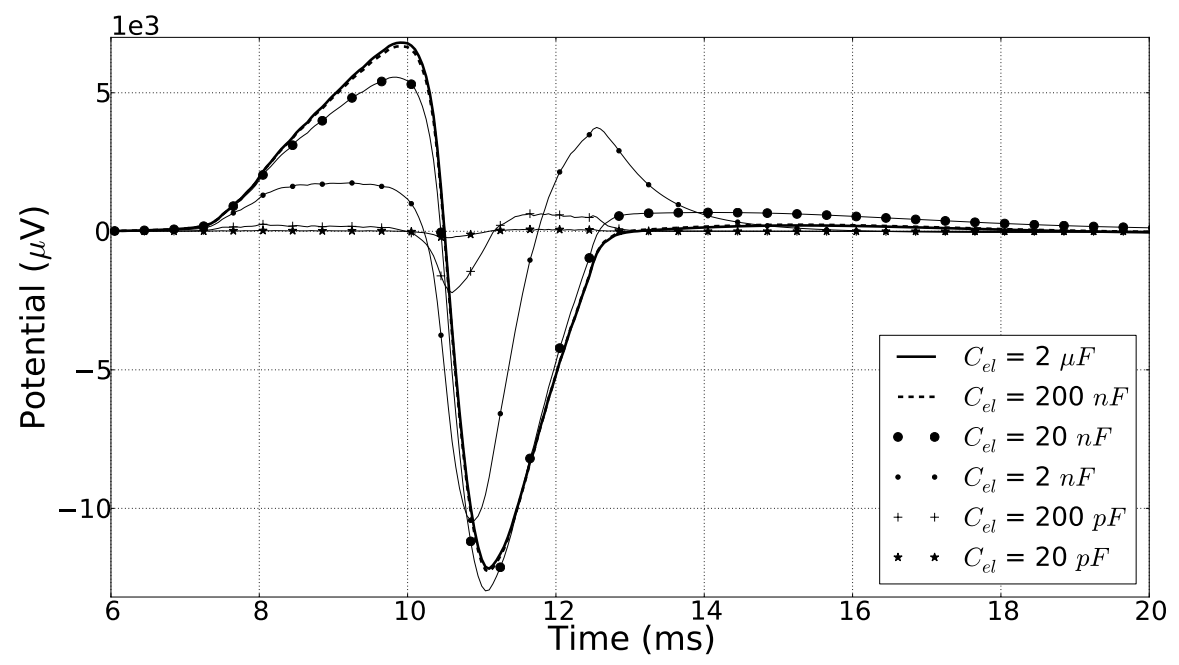

Figure 6: In silico depolarization phase of the field potential for different values of the electrode capacitance $C_{\mathrm{el}}$.

\subsection{Early afterdepolarization (EAD)}

For the applications in safety pharmacology, it is important to assess that our model is able to correctly reproduce the Field Potential observed in presence of 
an Early After Depolarizations (EADs, see for example [15]). To generate an EAD, we block $95 \%$ of $I_{K r}$ in the O'Hara-Rudy model [17], while all the other parameters of the model remain unchanged. The results are reported in Fig. 7.
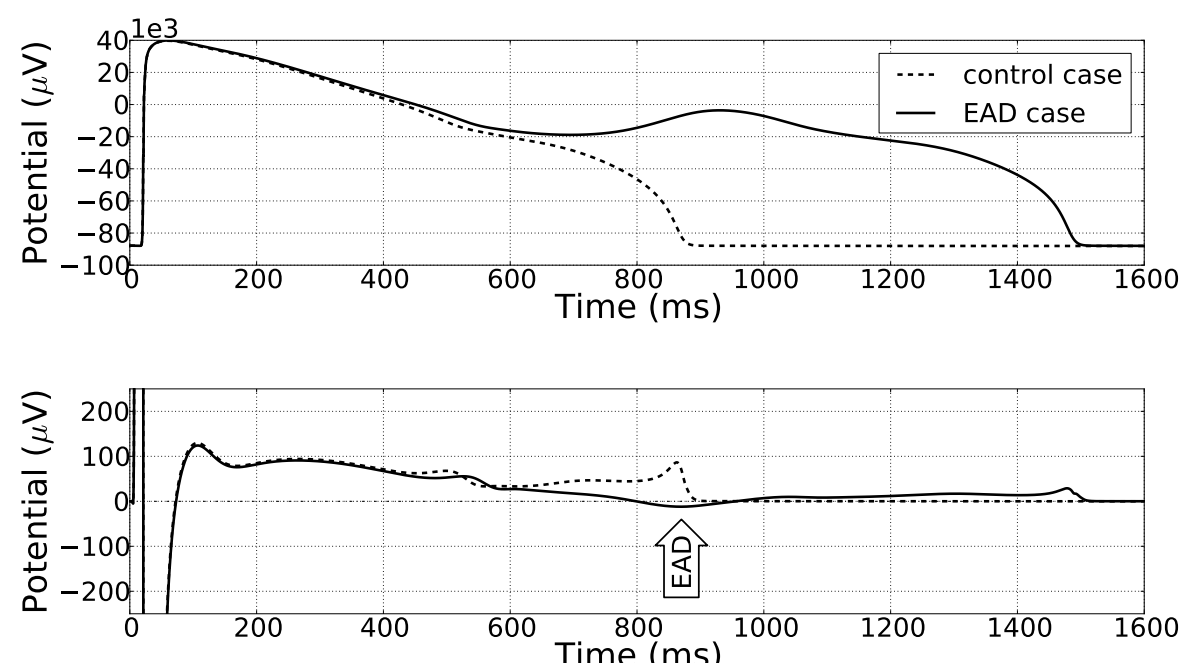

Figure 7: In silico transmembrane potential (top), field potential (bottom) corresponding to an EAD. Comparison with the control case (dotted line).

The AP behaves as expected (Fig. 7 top). The FP generated by our model exhibits a positive deflection in absence of $\mathrm{EAD}$ and a slightly negative deflection in presence of EAD (Fig. 7 bottom).

\subsection{Identification of conductances in the ionic current}

The results obtained for the identification of channel conductances are presented in this section. In paragraph 3.3.1, the algorithm presented in section 2.2 is verified by solving the identification problem in a situation where the results are known. To do so, synthetic measurements are first generated by numerical simulations. Then the channel conductances retrieved by the cost function optimization can be compared to the values used to generate the data. In paragraph 3.3.3, we use real in vitro experiments for different drugs and present the dose-response curves found by the algorithm.

For these simulations, the MV model was used. The ionic current is decomposed into three currents: the fast inward, slow inward and slow outward currents which, for simplicity, are assimilated to $\mathrm{Na}^{+}, \mathrm{Ca}^{2+}$ and $\mathrm{K}^{+}$respectively. 


\subsubsection{Verification with synthetic data}

The inverse problem strategy was tested to identify the conductances of the $\mathrm{Na}^{+}$ and $\mathrm{Ca}^{2+}$ channels from synthetic data. Numerical simulations are first used to generate the reference measurements represented by the continuous lines in Fig. 8. The black continuous line corresponds to the control case. The other continuous lines are obtained by partially blocking the activities of the $\mathrm{Na}^{+}$ and $\mathrm{Ca}^{2+}$ currents. The percentage of activity of $\mathrm{Na}^{+}$and $\mathrm{Ca}^{2+}$ are given in Table 1 . The activity was set to $100 \%$ in the control case.
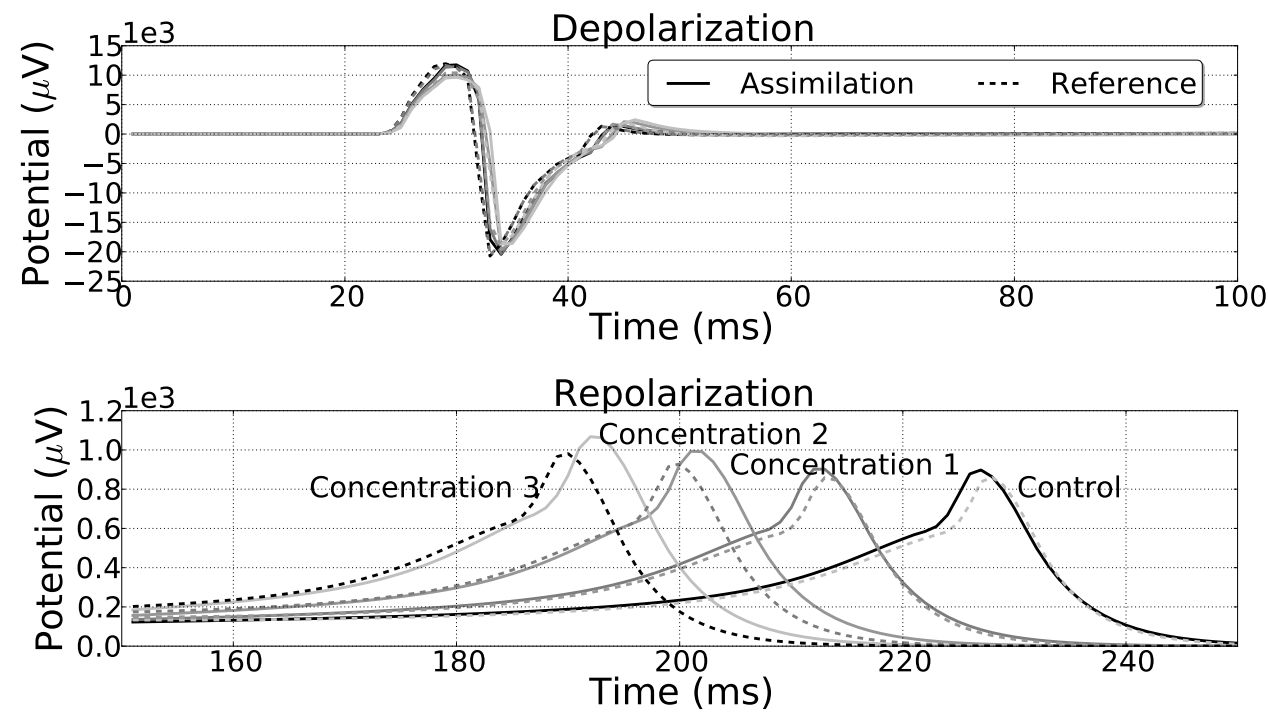

Figure 8: Depolarization and repolarization phases of the in silico field potentials in the assimilation process. The continuous lines correspond to the reference case and the dotted lines to the signal recovered by the assimilation process. The control case is represented in black and the curves are lighter when the activity of $\mathrm{Na}^{+}$and $\mathrm{Ca}^{2+}$ decreases (values are given in Table 1).

Then, for each concentration, we minimize the cost function (4) which involves the biomarkers DA, RA and FPD associated with the signals. To assess the stability of the process, we introduce a bias by choosing a set of model parameters different from the one used to generate these reference FPs.

The results of the assimilation process are given in the second column of Table 1 and the corresponding signals are represented by the dotted lines in Fig. 8. The last column of Table 1 contains the relative error between the reference values (the targets) and the values estimated by the algorithm. It can be observed that, for each measurement, the identification process succeeds in finding the reference values with a good accuracy. It is not surprising to 
obtain a non-vanishing discrepancy since a bias was introduced in the synthetic measurements.

\subsubsection{Influence of the number of electrodes}

\begin{tabular}{c|c|c|c} 
& Target & Estimation & Relative error (in \%) \\
\hline Case 1 & $(96,92)$ & $(94,93)$ & 1.7 \\
\hline Case 2 & $(78,85)$ & $(78,88)$ & 2.6 \\
\hline Case 3 & $(71,80)$ & $(73,85)$ & 4.8
\end{tabular}

Table 1: Estimation of the activity of the $\mathrm{Na}^{+}$and $\mathrm{Ca}^{2+}$ channels (the control case corresponds to an activity equal to $100 \%$ ).

In the resolution of the inverse problem, we have used the FP signals collected at a given number of electrodes. It is of practical interest to wonder if it is important to take several electrodes and if the choice of the electrodes has an impact on the accuracy of the results given by our identification process.

In Fig. 9, the relative error between the value found by the identification process and the reference value is presented for different electrode selections. These tests have been made with the cost function (3) which involves the whole FP. We observe that the relative error largely varies from case to case. Even if the error may be small with only one or two electrodes, this performance depends on the selection of the electrodes, and that itself depends on the location of the initial activation, which is a priori unknown. Thus, these tests suggest that it is safer to use all the measurements collected by the electrodes.

\subsubsection{Tests with experimental measurements}

We now consider in vitro FPs recorded in the presence of different drugs. All the data were provided by Janssen Pharmaceutica NV using MC_Rack (Multi Channel Systems GmbH) and post-processed by NOTOCORD Systems (NOTOCORD-FPS 3.0 software). The cells were produced by Cellular Dynamics International (type iCell ${ }^{\circledR}$ Cardiomyocytes, 2012). Fig. 10 shows an example of FPs recorded at the 9 microelectrodes of a 6-well MEA. Another illustration of in vitro FPs is given in Fig. 11 which shows FPs recorded for different concentrations of moxifloxacin.

Our objective is to identify the activity of the $\mathrm{Na}^{+}, \mathrm{K}^{+}$and $\mathrm{Ca}^{2+}$ channels for each drug concentration. To achieve this goal, the cost function (4), which measures the discrepancy between the biomarkers, was minimized. For the control case and different drug concentrations, the biomarkers DA, RA and FPD associated with the mean FP are extracted at each electrode of the MEA and these data are used as the reference biomarkers in the cost function (4).

In the following paragraphs, the effects of moxifloxacin, ivabradine, SEA0400 and diltiazem on FPs are presented. It was observed that a blockage of the $\mathrm{K}^{+}$ 
$g_{f i}$ assimilation with noised observations

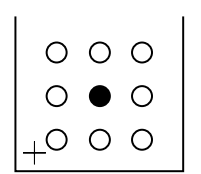

Error: $18.25 \%$

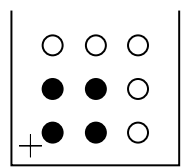

Error: $7.50 \%$

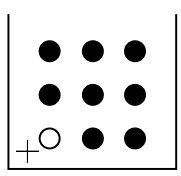

Error: $2.40 \%$

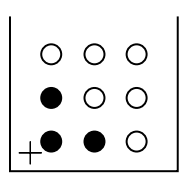

Error: $14.75 \%$

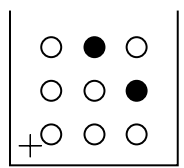

Error: $3.50 \%$

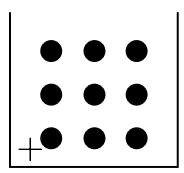

Error: $2.23 \%$

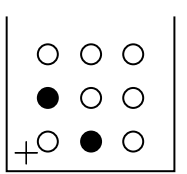

Error: $13.50 \%$

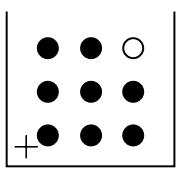

Error: $3.50 \%$

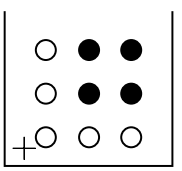

Error: $1.75 \%$

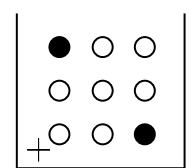

Error: $7.75 \%$

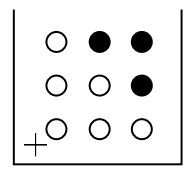

Error: $3.25 \%$

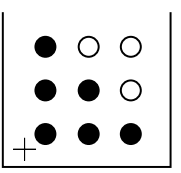

Error: $0.75 \%$

Observation

Figure 9: For each well, only the measurements given by the electrodes represented by the continuous black points are used in the identification algorithm. The location of the initial stimulation is represented by a cross. The error corresponds to the relative error between the reference values and the values given by the identification algorithm.

channel induced an increase in the FPD, while a blockage of the $\mathrm{Ca}^{2+}$ induced a decrease in the FPD. Based on this simple observation, we decided to fix the value of the conductance of one of those two channels. This manual preprocessing simplifies the task of the optimization algorithm and limits identifiability issues. Following this guideline, and considering the experimental FPD evolution, the activity of the $\mathrm{Ca}^{2+}$ channel was fixed for moxifloxacin and ivabradine, as was fixed the activity of the $\mathrm{K}^{+}$channel for diltiazem and SEA0400.

The results of the optimization process are summarized in the dose-response curves depicted in Figures 12, 13, 14 and 15 which show the ion channel relative conductances under the action of the four different drugs.

For moxifloxacin and ivabradine, which are known to be potassium blockers, the algorithm correctly captures the effect of the drug, not only qualitatively but also quantitatively. Indeed, the dose-response curve obtained by parameter identification is in good agreement with the theoretical dose-response curve, represented in dotted lines in Figures 12 and 13. The theoretical curve is given 


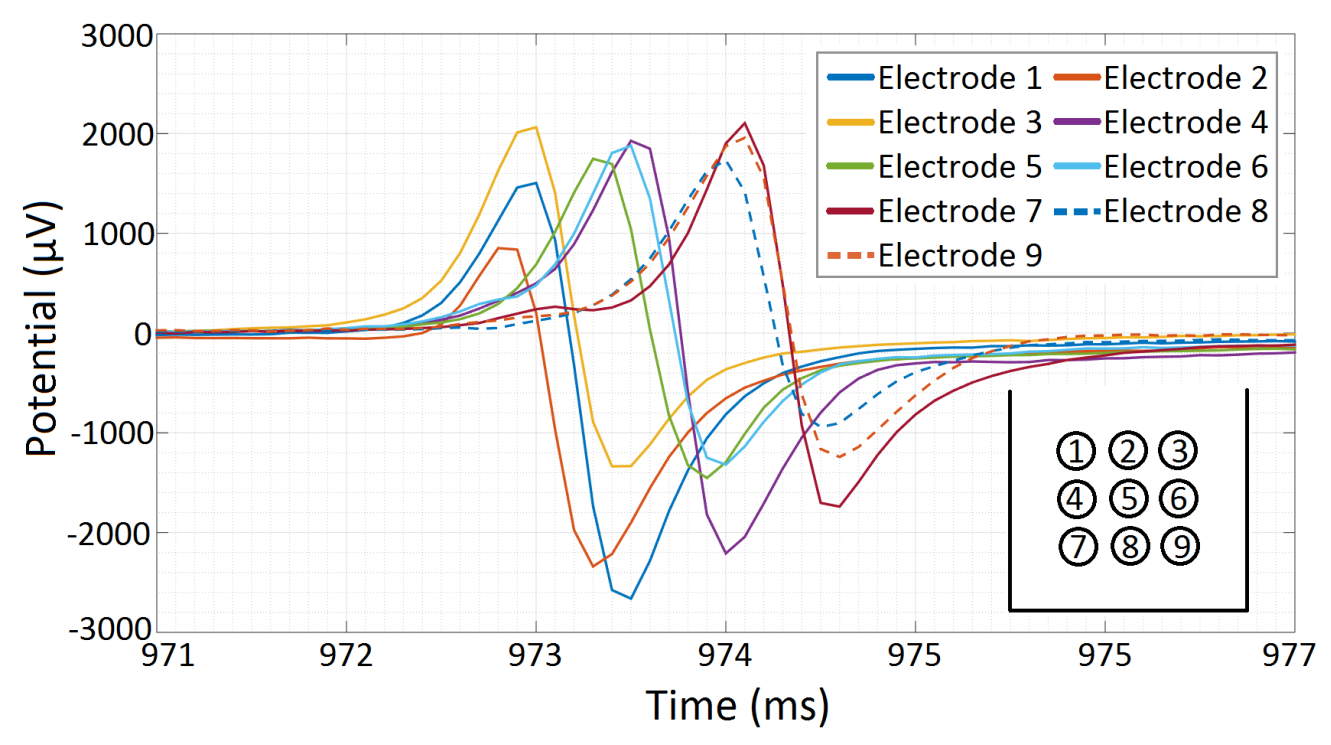

Figure 10: Example of in vitro FPs recorded (depolarization phase).

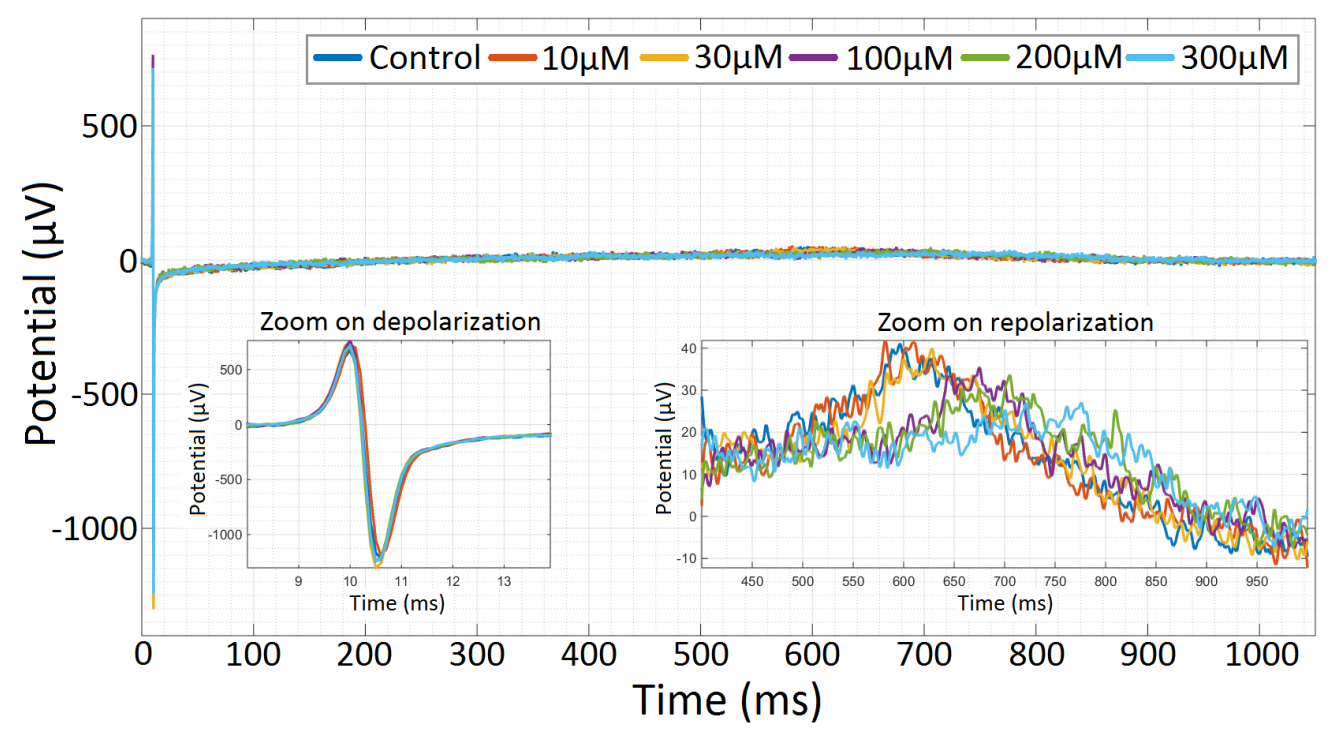

Figure 11: Effects of moxifloxacin on in vitro FPs recorded at different concentrations.

by

$$
\left(1+\frac{[c]}{I C_{50}}\right)^{-1}
$$




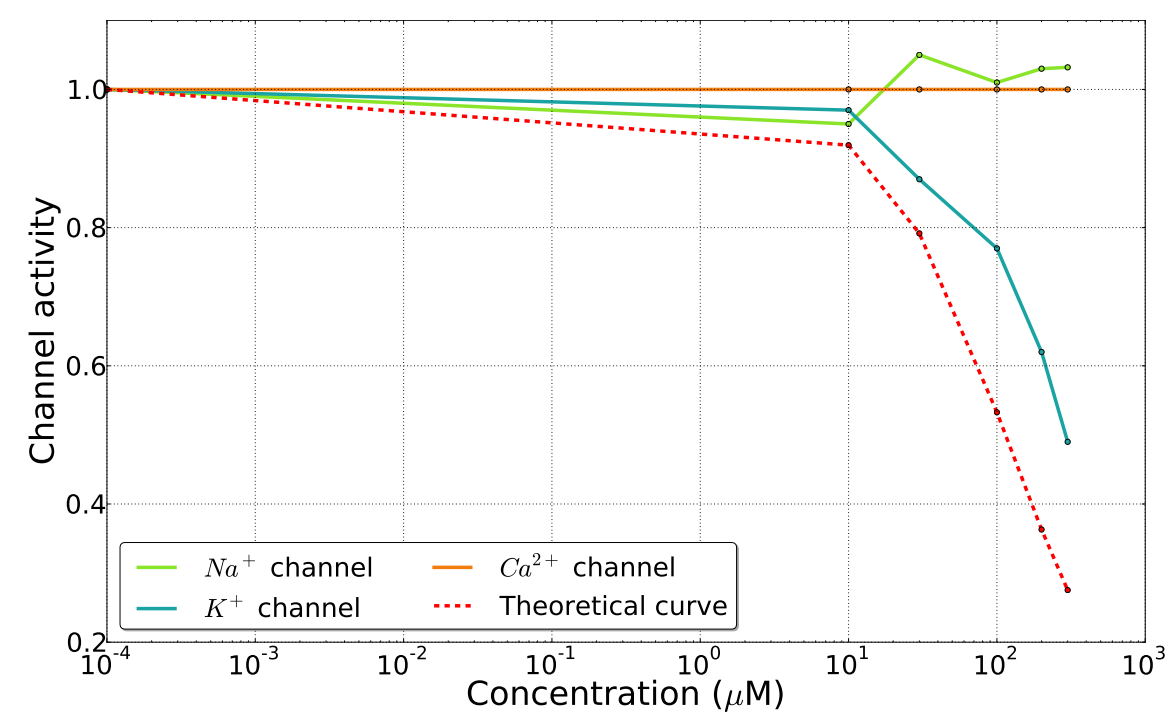

Figure 12: Moxifloxacin effect on each ion channel activity (in vitro data).

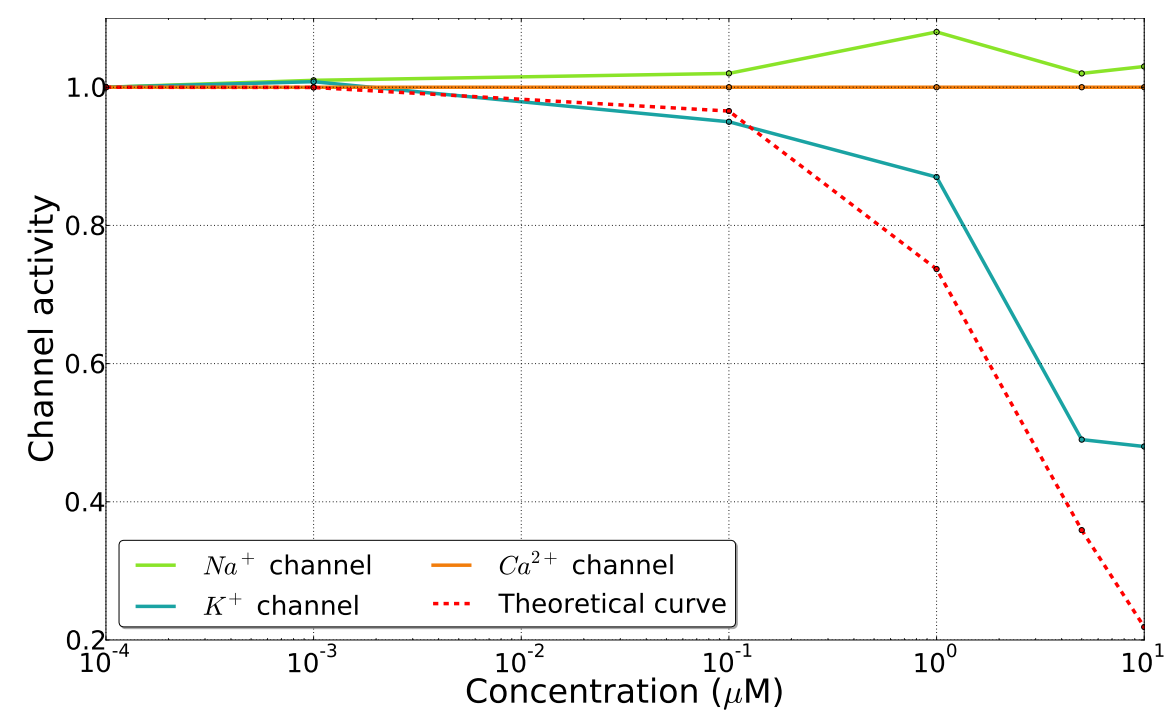

Figure 13: Ivabradine effects on each ion channel activity (in vitro data).

where the values of $I C_{50}$ of the current $I_{K}$ are taken from the literature: for moxifloxacin, $I C_{50}=114 \mu M$ [2] and for ivabradine, $I C_{50}=2.07 \mu M$ [16].

SEA0400 is known to be a Na-Ca exchanger blocker and it has also been shown to be L-type calcium channel blocker above $1 \mu M$ [19]. Fig. 14 shows that 


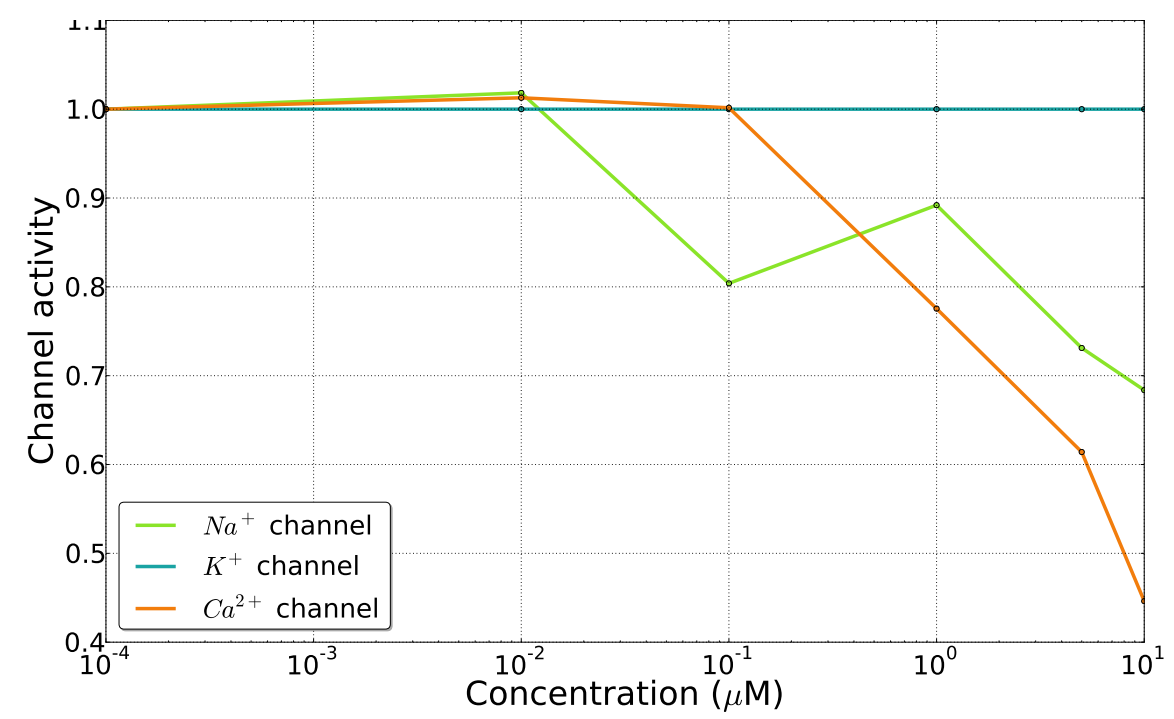

Figure 14: SEA0400 effects on each ion channel activity (in vitro data).

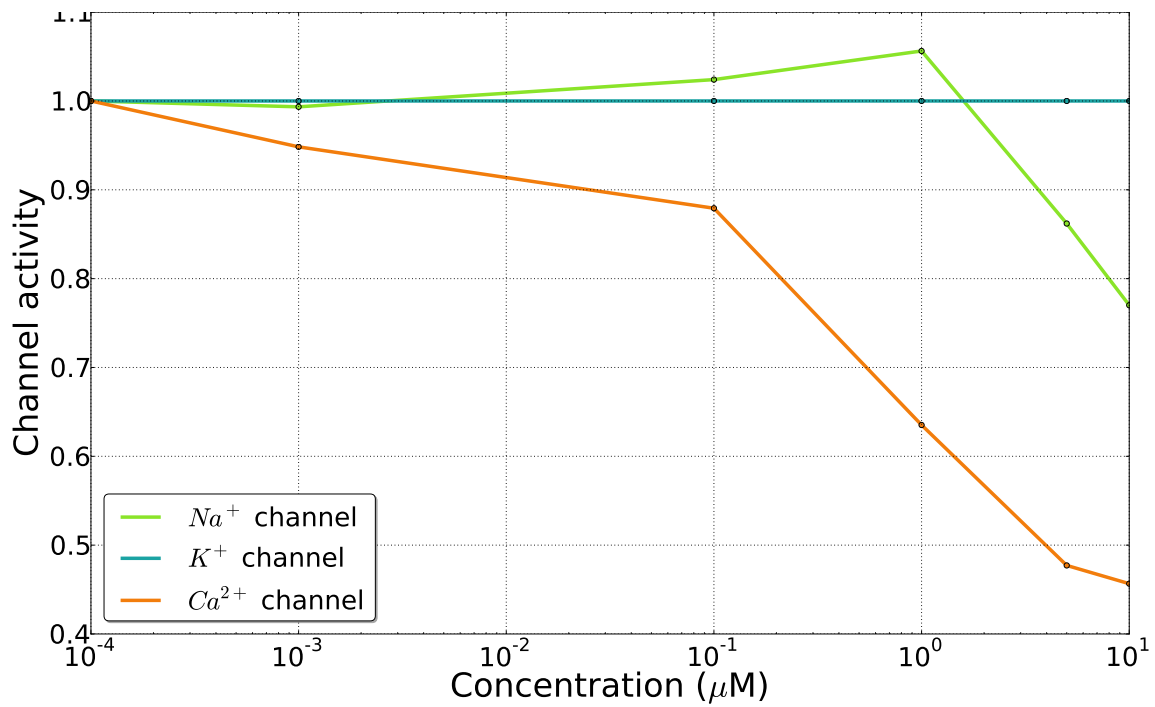

Figure 15: Diltiazem effects on each ion channel activity (in vitro data).

the algorithm succeeds in detecting that $\mathrm{Na}^{+}$and $\mathrm{Ca}^{2+}$ channels are affected under the action of this compound.

Fig. 15 shows that the algorithm detects an impact of diltiazem on the $\mathrm{Ca}^{2+}$ channel, but also on the $\mathrm{Na}^{+}$channel. These results are concordant with the 
well-established calcium antagonist properties of diltiazem [10], as well with those suggesting that diltiazem affect Nav1.5 channel activity at high concentrations [13].

As an example, Table 2 shows in the case of moxifloxacin the results achieved by the optimization process for the five concentrations used for the data represented in Fig. 11. The discrepancy between the experimental biomarkers and the biomarkers found at the end of the parameter identification process is generally less than $10 \%$. Similar results are obtained for diltiazem, ivabradine and SEA0400 (Suppl. Material S4).

\begin{tabular}{|l|c|c|c|c|c|c|}
\hline & $\begin{array}{c}\text { DA ratio } \\
\text { (simulation) }\end{array}$ & $\begin{array}{c}\text { DA ratio } \\
\text { (experimental) }\end{array}$ & $\begin{array}{c}\text { RA ratio } \\
\text { (simulation) }\end{array}$ & $\begin{array}{c}\text { RA ratio } \\
\text { (experimental) }\end{array}$ & $\begin{array}{c}\text { FPD ratio } \\
\text { (simulation) }\end{array}$ & $\begin{array}{c}\text { FPD ratio } \\
\text { (experimental) }\end{array}$ \\
\hline Concentration 1 & 0,98 & 0.98 & 1,00 & 1,00 & 1,00 & 1,01 \\
\hline Concentration 2 & 1,09 & 1.08 & 0,90 & 0,89 & 1,04 & 1,04 \\
\hline Concentration 3 & 1,00 & 1.00 & 0,86 & 0,80 & 1,17 & 1,13 \\
\hline Concentration 4 & 1,03 & 1.02 & 0,77 & 0,71 & 1,30 & 1,20 \\
\hline Concentration 5 & 1,15 & 1.03 & 0,71 & 0,63 & 1,42 & 1,31 \\
\hline
\end{tabular}

Table 2: Experimental and numerical biomarkers after the identification process for moxifloxacin. The good agreement shows that the cost function reaches a low value.

We report the whole in silico signals obtained at the end of the identification process in the Supplementary Materials S5. We also show results of the identification using the Paci model (Supplementary Material S6).

\section{Discussion}

In silico approaches of MEA measurements are not very common in the literature. The first goal of this study was, therefore, to show that a mathematical model is actually able to provide realistic synthetic signals. The results obtained for two commercial devices (Fig. 2 and 3) are in a good qualitative agreement with the signals observed in reality. In the simulation of a FP during an EAD (Fig. 7), the deflection may be difficult to identify if considered alone, but it is clearly visible if compared to the case without EAD (dashed line in Fig. 7). Besides, this kind of slight deflections is in good agreement with MEA experimental measurements of EADs reported in the literature (see e.g. Fig. 5 (D) in [3]).

The strong variability of experimental signals did not allow us to make a more quantitative validation. Nevertheless, the results obtained with our strategy of parameter identification show that this level of realism seems to be sufficient to address questions of practical relevance.

An appealing feature of the in silico approach is its capability to isolate phenomenon and to test hypotheses more easily than in real experiments. For 
example, it is not straightforward to experimentally understand the variability observed in the FP within a MEA well. A classical explanation is the heterogeneity of the hiPSC-CM population [8]. In this work, we showed in silico that FP variations appear even with a homogeneous population of cells (Figures 3 and 4). Our simulations showed that these variations, both in amplitude and in orientation, are linked to different geometrical factors: the size of the MEA, the inter-electrode distance, the position of the ground and the relative positions of the measurement electrode and the initial activation. Thus, the electrode-toelectrode variability of the FP is dependent on the type of MEA device.

The identification of channel activities from FP measurements is the most practical result of the present work. We showed that, even when the model does not quantitatively reproduce the real signal, it is still possible to automatically detect which channel is affected by renormalizing the biomarkers with a control case. Thus it is not necessary to perfectly know all the parameters of the model (electrical conductivities, location of the original activation, etc.), as soon as those parameters do not significantly affect the drug-induced evolution of the biomarkers with respect to their control values. Our strategy allowed us to estimate the dose-response curve from four experimental datasets. For moxifloxacin and ivabradine, the algorithm was even able to recover the IC50 with a good accuracy.

In spite of these promising results, the limitations of the approach are the following:

(i) The choice of the bidomain equations to describe the diffusion of the potential in the MEA can be criticized since there is no clear scale separation between the size of the electrode and the size of the cells. An alternative would consist of an individual description of each cell. This would be much more demanding from a computational viewpoint and this would certainly raise many other modeling issues. That is why the approximation of a homogenized medium was regarded as a starting point. The obtained results seem to validate this choice a posteriori.

(ii) The ionic model is also a critical ingredient. The Paci model was specifically designed to describe hiPSC-CM and should therefore be firstly selected. But, at the moment, there is still a strong variability in the stem cells produced by different manufacturers. Besides, like the O'Hara-Rudy model, it relies on many parameters which are difficult, or even impossible, to identify from the FP. The choice to solve the inverse problem with the MV model is, therefore, a compromise between realism and identifiability issues. We have nevertheless checked that it was also possible to solve an inverse problem with the Paci model (Supplementary Materials).

In Fig. 12-15, it can be observed that the activity of the sodium channel is not monotonic with respect to the concentration. The reason for this strange behavior is probably the high sensitivity of the fast inward conductance of the MV model with respect to the depolarization amplitude. This point deserves a deeper investigation, in particular with other compounds and other ionic models.

It should also be noted that the equations depend on several parameters which are essentially unknown: $A_{m}, C_{m}, R_{e l}, R_{i}, C_{e l}, \sigma_{\mathrm{i}}, \sigma_{\mathrm{e}}, z_{t h i c k}$, location 
of the initial activation, etc. Some of them could be estimated by direct measurements (e.g. [9]). This was not done in this study. Instead, their values were fixed to recover the main features of the experimental data (global shape, propagation velocity). With the strategy consisting in measuring the evolution of the biomarkers with respect to a control value, this choice was shown to be sufficient.

\section{Conclusion}

MEAs, combined with hiPSC-CMs, can enable high- or medium-throughput drug screening in safety pharmacology. But this promising technology is currently limited by the difficulty to analyze the measured FP. In the present work, we proposed a strategy to analyze the FP acquired by the MEA and to automatically deduce the ionic channels affected by a compound. The method relies on a mathematical model of the cells in the MEA, and on an inverse problem algorithm. We first showed that the model was able to reproduce features actually observed in real experiments. Then, we tested our strategy on real data and we were able to identify the channels affected by four compounds.

Many aspects of our strategy could be improved: ionic models tailored to specific stem cells could be considered; other biomarkers could be introduced to limit the identifiability issues; a more systematic way to set the model parameters could be devised; our approach could be combined with other modalities, like fluorescence. Besides, the preliminary results presented in this article have to be confirmed with more compounds. This is the topic of current investigations.

To conclude, it should be noticed that pharmacological tests are much easier to perform with MEAs than with more traditional techniques. This allows a huge quantity of data to be acquired. MEAs measurements will therefore not only benefits from in silico approaches, but they will also certainly contribute to improve them.

\section{Acknowledgments}

This work was partially supported by the French National Agency of Research through the grant ANR-13-LAB1-0007 (LabCom "CardioXcomp"), by Instem, and by the Agency for Interaction in Mathematics with Business and Society (AMIES). We would like to thank Janssen Pharmaceutica NV for providing us with the raw experimental data.

\section{References}

[1] E. Abbate, M. Boulakia, Y. Coudière, J-F. Gerbeau, P. Zitoun, and $\mathrm{N}$. Zemzemi. In silico assessment of the effects of various compounds in 
MEA/hiPSC-CM assays: Modelling and numerical simulations. Research report, Inria, https://hal.inria.fr/hal-01562673, July 2017.

[2] A.J. Alexandrou, R.S. Duncan, A. Sullivan, J.C. Hancox, D.J. Leishman, H.J. Witchel, and J.L. Leaney. Mechanism of hERG K+ channel blockade by the fluoroquinolone antibiotic moxifloxacin. British Journal of Pharmacology, 147(8):905-916, 2006.

[3] K. Asakura, S. Hayashi, A. Ojima, T. Taniguchi, N. Miyamoto, C. Nakamori, C. Nagasawa, T. Kitamura, T. Osada, Y. Honda, C. Kasai, H. Ando, Y. Kanda, Y. Sekino, and K. Sawada. Improvement of acquisition and analysis methods in multi-electrode array experiments with iPS cellderived cardiomyocytes. J. Pharmacol. Toxicol. Methods, 75:17-26, 2015.

[4] A. Bueno-Orovio, E.M. Cherry, and F.H. Fenton. Minimal model for human ventricular action potentials in tissue. J. Theor. Biol., 253(3):544-560, 2008.

[5] I. Cavero, J.-M. Guillon, V. Ballet, M. Clements, J.-F. Gerbeau, and H. Holzgrefe. Comprehensive in vitro Proarrhythmia Assay (CiPA): Pending issues for successful validation and implementation. Journal of Pharmacological and Toxicological Methods, 2016.

[6] I. Cavero and H. Holzgrefe. Cipa: Ongoing testing, future qualification procedures, and pending issues. Journal of pharmacological and toxicological methods, 76:27-37, 2015.

[7] R.H. Clayton and A.V. Panfilov. A guide to modelling cardiac electrical activity in anatomically detailed ventricles. Progress in Biophysics and Molecular Biology, 96:19-43, 2008.

[8] M.X. Doss, J.M. Di Diego, R. Goodrow, Y. Wu, J.M. Cordeiro, V. Nesterenko, H. Barajas-Martínez, D. Hu, J. Urrutia, M. Desai, and C. Antzelevitch. Maximum diastolic potential of human induced pluripotent stem cell-derived cardiomyocytes depends critically on ikr. PloS one, 7(7):e40288, 2012.

[9] E. Gilboa, P.S. La Rosa, and A. Nehorai. Estimating electrical conductivity tensors of biological tissues using microelectrode arrays. Annals of biomedical engineering, 40(10):2140-2155, 2012.

[10] T. Godfraind, R. Miller, and M. Wibo. Calcium antagonism and calcium entry blockade. Pharmacological Reviews, 38(4):321-416, 1986.

[11] N. Hansen and A. Ostermeier. Completely derandomized self-adaptation in evolution strategies. Evolutionary Computation, 9(2):159-195, 2001.

[12] A.C. Hindmarsh, P.N. Brown, K.E. Grant, S.L. Lee, R. Serban, D.E. Shumaker, and C.S. Woodward. Sundials: Suite of nonlinear and differential/algebraic equation solvers. ACM Transactions on Mathematical Software (TOMS), 31(3):363-396, 2005. 
[13] C. Hirth, U. Borchard, and D. Hafner. Effects of the calcium antagonist diltiazem on action potentials, slow response and force of contraction in different cardiac tissues. Journal of molecular and cellular cardiology, 15(12):799-809, 1983.

[14] J. Huo, A. Kamalakar, X. Yang, B. Word, N. Stockbridge, B. Lyn-Cook, and L. Pang. Evaluation of batch variations in induced pluripotent stem cell-derived human cardiomyocytes from 2 major suppliers. Toxicological Sciences, page kfw235, 2016.

[15] P. Kügler. Early afterdepolarizations with growing amplitudes via delayed subcritical hopf bifurcations and unstable manifolds of saddle foci in cardiac action potential dynamics. PloS one, 11(3):e0151178, 2016.

[16] D. Melgari, K.E. Brack, C. Zhang, Y. Zhang, A. El Harchi, J.S. Mitcheson, C.E. Dempsey, G.A. Ng, and J.C. Hancox. hERG potassium channel blockade by the HCN channel inhibitor bradycardic agent ivabradine. Journal of the American Heart Association, 4(4):e001813, 2015.

[17] T. O'Hara, L. Virag, A. Varro, and Y. Rudy. Simulation of the undiseased human cardiac ventricular action potential: Model formulation and experimental validation. PLoS Computational Biology, 7(5):1-29, 2011. doi: 10.1371/journal.pcbi.1002061.

[18] M. Paci, J. Hyttinen, K. Aalto-Setala, and S. Severi. Computational models of ventricular- and atrial-like human induced pluripotent stem cell derived cardiomyocytes. Ann. Biomed. Eng., 41(11):2334-2348, 2013.

[19] H. Tanaka, K. Nishimaru, T. Aikawa, W. Hirayama, Y. Tanaka, and K. Shigenobu. Effect of SEA0400, a novel inhibitor of sodium-calcium exchanger, on myocardial ionic currents. British Journal of Pharmacology, 135(5):1096-1100, 2002.

[20] K.H. ten Tusscher, D. Noble, P.J. Noble, and A.V. Panfilov. A model for human ventricular tissue. American Journal of Physiology-Heart and Circulatory Physiology, 286(4):H1573-H1589, 2004.

[21] L. Tung. A bi-domain model for describing ischemic myocardial $D-C$ potentials. PhD thesis, MIT, 1978. 


\section{SUPPLEMENTARY MATERIAL}

\section{S1 Bidomain equations}

Our mathematical model of MEA is based on the bidomain equations which describe the evolution of the transmembrane potential $V_{\mathrm{m}}$ and the extracellular potential $\phi_{\mathrm{e}}$ through two coupled partial differential equations:

$$
\left\{\begin{array}{l}
A_{\mathrm{m}} C_{\mathrm{m}} \frac{\partial V_{\mathrm{m}}}{\partial t}+A_{\mathrm{m}} I_{\mathrm{ion}}\left(V_{m}, w\right)-\nabla \cdot\left(\sigma_{\mathrm{i}} \nabla V_{\mathrm{m}}\right)- \\
\nabla \cdot\left(\sigma_{\mathrm{i}} \nabla \phi_{\mathrm{e}}\right)=A_{\mathrm{m}} I_{\mathrm{app}} \\
-\nabla \cdot\left(\left(\sigma_{\mathrm{i}}+\sigma_{\mathrm{e}}\right) \nabla \phi_{\mathrm{e}}\right)-\nabla \cdot\left(\sigma_{\mathrm{i}} \nabla V_{\mathrm{m}}\right)=\frac{1}{z_{\text {thick }}} \sum_{e_{k}} \frac{I_{\mathrm{el}}^{k}}{\left|e_{k}\right|} \chi_{e_{k}},
\end{array}\right.
$$

where $A_{\mathrm{m}}$ is the surface area of membrane per unit volume of tissue, $C_{\mathrm{m}}$ is the membrane capacitance, $I_{\text {app }}$ is the current applied in the stimulation area, $\sigma_{\mathrm{i}}$ and $\sigma_{\mathrm{e}}$ correspond respectively to the intra- and extracellular conductivity tensors and $z_{\text {thick }}$ is the thickness of the cell layer. The ionic current $I_{\text {ion }}$ is provided by an ionic model. The signal is triggered by the stimulation current $I_{\text {app }}$, applied in an arbitrary zone of the MEA surface. The right-hand side of the second equation is due to the presence of the electrodes. In this expression, $I_{\mathrm{el}}^{k}$ is the electric current which goes through the electrode located at $e_{k},\left|e_{k}\right|$ is the electrode surface and $\chi_{e_{k}}$ is the characteristic function of $e_{k}$ (which takes the value 1 on the electrode and 0 elsewhere). The expression of $I_{\mathrm{el}}^{k}$ is given in the following paragraph. Let $\boldsymbol{n}$ be the outward normal to the boundary of the domain. Equations (6) are completed with the following boundary conditions: $\sigma_{\mathrm{i}} \nabla \phi_{\mathrm{i}} \cdot \boldsymbol{n}=0$ (where $\phi_{\mathrm{i}}=V_{\mathrm{m}}+\phi_{\mathrm{e}}$ ), and either $\phi_{e}=0$ on the region connected to the ground or $\sigma_{\mathrm{e}} \nabla \phi_{\mathrm{e}} \cdot \boldsymbol{n}=0$ elsewhere.

\section{S2 Geometrical modeling of two commercial de- vices}

In the 96-well MEA, the disc-shaped well is made of 8 microelectrodes and 4 grounds located around the electrodes (Figure S1 top). In the 6-well MEA, the square-shaped well is made of 9 microelectrodes and a ground which is located at three sides of the square (Figure S1 bottom). Figure S1 shows the finite element meshes used in the numerical resolution.

\section{S3 Relationship between FPD and APD}

In reference [3], K. Asakura et al. measured simultaneously APD with voltagesensitive dye and FPD in a dish-shaped well of a Multi Electrode Array of type MED64. They found that FPD recorded was roughly equal to APD50. As a first approximation, our simulations are not far from this observation. 

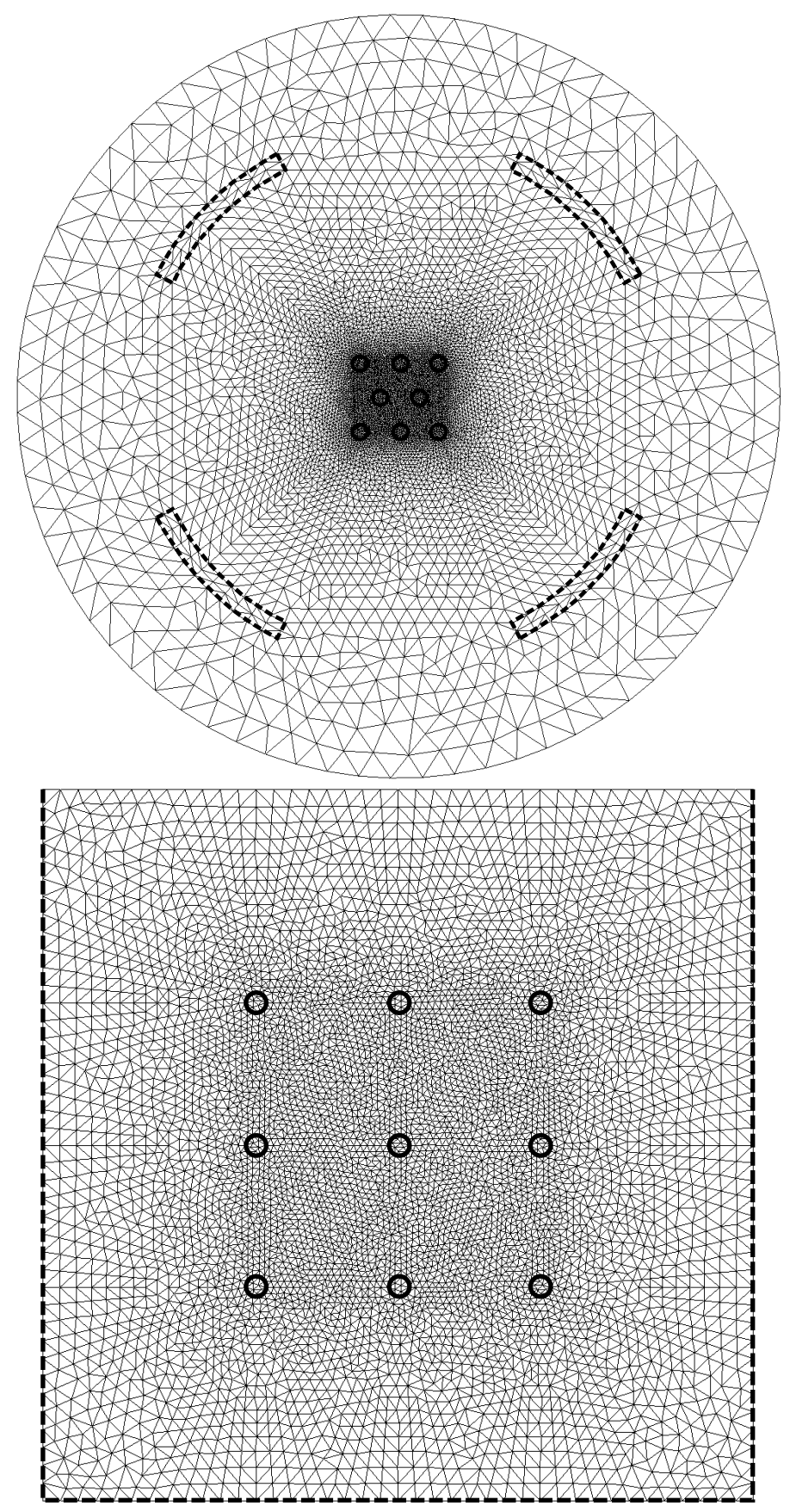

Figure S1: Geometry of the MEA wells and the finite element meshes: discshaped well with 8 microelectrodes in the 96-well MEA (top), square-shaped well with 9 microelectrodes in the 6 -well MEA (bottom). The electrodes are represented by the small rings and the grounds by the dotted lines. 
But more in details, Figures S2 and S3 show that the relationship between the simulated FDP and APD seems to depend on the device, on the drug concentration, and on the electrodes. In the 8-electrode MEA, the FPD corresponds approximatively to the APD65 (Figure S2) whereas in the 9-electrode MEA it is about APD45 (Figure S2).

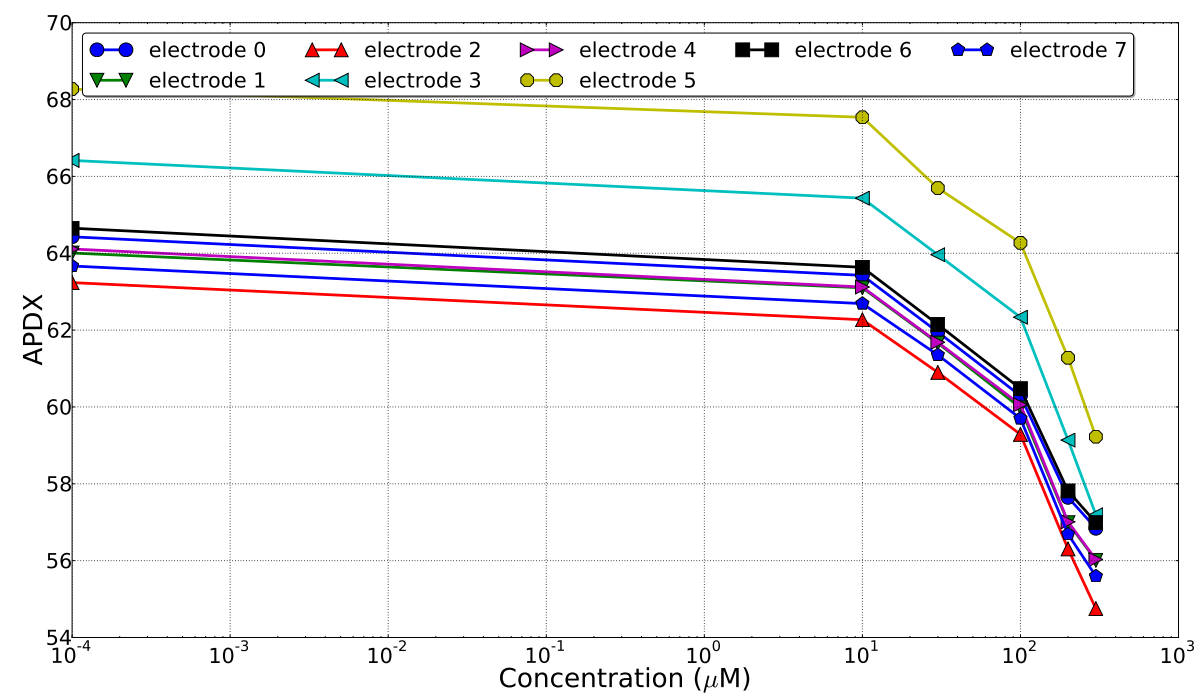

Figure S2: Comparison between FPD and APD using results from Moxifloxacin parameter identification (MEA with 8 electrodes per well).

\section{S4 Experimental and numerical biomarkers af- ter identification}

Experimental and numerical biomarkers after the identification process are presented for diltiazem (Table S1), ivabradine (Table S2) and SEA0400 (Table S3).

\section{S5 In silico Field Potentials after identification}

As explained in Sections II.B and III.C, when dealing with real data, the inverse problem is solved by reducing the misfit between ratios of biomarkers. The relevant quantities to compare at the end of the identification process are therefore those presented in Table II of the article. Nevertheless, it is legitimate to wonder what is the shape of the full numerical FP signal after identification. This is shown in Figure S4 in the case of moxifloxacin. Comparing this figure with the experimental FPs displayed in Fig. 11 of the article, we observe that the in silico 


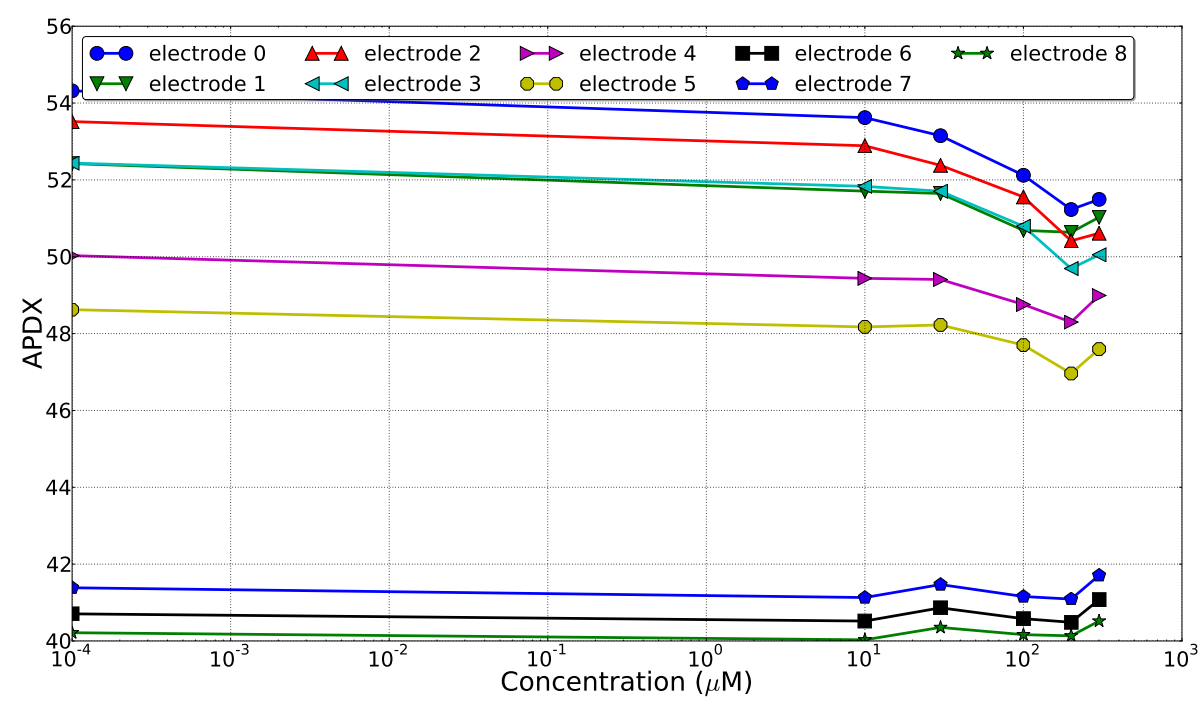

Figure S3: Comparison between FPD and APD using results from Moxifloxacin parameter identification (MEA with 9 electrodes per well).

\begin{tabular}{|l|c|c|c|c|c|c|}
\hline & $\begin{array}{c}\text { DA ratio } \\
\text { (simulation) }\end{array}$ & $\begin{array}{c}\text { DA ratio } \\
\text { (experimental) }\end{array}$ & $\begin{array}{c}\text { RA ratio } \\
\text { (simulation) }\end{array}$ & $\begin{array}{c}\text { RA ratio } \\
\text { (experimental) }\end{array}$ & $\begin{array}{c}\text { FPD ratio } \\
\text { (simulation) }\end{array}$ & $\begin{array}{c}\text { FPD ratio } \\
\text { (experimental) }\end{array}$ \\
\hline Concentration 1 & 0,98 & 0,99 & 1,00 & 0,98 & 0,96 & 0,97 \\
\hline Concentration 2 & 1,01 & 1,02 & 0,98 & 0,96 & 0,91 & 0,93 \\
\hline Concentration 3 & 1,04 & 1,05 & 0,88 & 0,85 & 0,69 & 0,71 \\
\hline Concentration 4 & 0,88 & 0,80 & 0,62 & 0,45 & 0,47 & 0,53 \\
\hline Concentration 5 & 0,82 & 0,78 & 0,60 & 0,45 & 0,45 & 0,52 \\
\hline
\end{tabular}

Table S1: Ratio comparison after parameter identification using diltiazem data as observation.

\begin{tabular}{|l|c|c|c|c|c|c|}
\hline & $\begin{array}{c}\text { DA ratio } \\
\text { (simulation) }\end{array}$ & $\begin{array}{c}\text { DA ratio } \\
\text { (experimental) }\end{array}$ & $\begin{array}{c}\text { RA ratio } \\
\text { (simulation) }\end{array}$ & $\begin{array}{c}\text { RA ratio } \\
\text { (experimental) }\end{array}$ & $\begin{array}{c}\text { FPD ratio } \\
\text { (simulation) }\end{array}$ & $\begin{array}{c}\text { FPD ratio } \\
\text { (experimental) }\end{array}$ \\
\hline Concentration 1 & 1,01 & 1,00 & 1,00 & 0,99 & 0,99 & 1,01 \\
\hline Concentration 2 & 1,04 & 1,03 & 0,96 & 0,97 & 1,04 & 1,07 \\
\hline Concentration 3 & 1,09 & 1,11 & 0,83 & 0,84 & 1,24 & 1,36 \\
\hline Concentration 4 & 1,04 & 1,02 & 0,75 & 0,80 & 1,41 & 1,43 \\
\hline Concentration 5 & 1,02 & 1,00 & 0,53 & 0,58 & 1,65 & 1,99 \\
\hline
\end{tabular}

Table S2: Ratio comparison after parameter identification using ivabradine data as observation. 


\begin{tabular}{|l|c|c|c|c|c|c|}
\hline & $\begin{array}{c}\text { DA ratio } \\
\text { (simulation) }\end{array}$ & $\begin{array}{c}\text { DA ratio } \\
\text { (experimental) }\end{array}$ & $\begin{array}{c}\text { RA ratio } \\
\text { (simulation) }\end{array}$ & $\begin{array}{c}\text { RA ratio } \\
\text { (experimental) }\end{array}$ & $\begin{array}{c}\text { FPD ratio } \\
\text { (simulation) }\end{array}$ & $\begin{array}{c}\text { FPD ratio } \\
\text { (experimental) }\end{array}$ \\
\hline Concentration 1 & 1,04 & 1,03 & 1,00 & 1,00 & 1,01 & 1,00 \\
\hline Concentration 2 & 0,84 & 0,70 & 1,00 & 0,96 & 1,00 & 1,00 \\
\hline Concentration 3 & 0,87 & 0,78 & 0,95 & 0,88 & 0,83 & 0,82 \\
\hline Concentration 4 & 0,92 & 0,85 & 0,84 & 0,81 & 0,65 & 0,73 \\
\hline Concentration 5 & 0,75 & 0,68 & 0,67 & 0,53 & 0,27 & 0,27 \\
\hline
\end{tabular}

Table S3: Ratio comparison after parameter identification using SEA0400 data as observation.

FP signals capture the main trends of the experimental FPs. It is nevertheless clear that the two signals cannot be superimposed, which is consistent with our choice of comparing biomarkers ratios rather than the full signal.

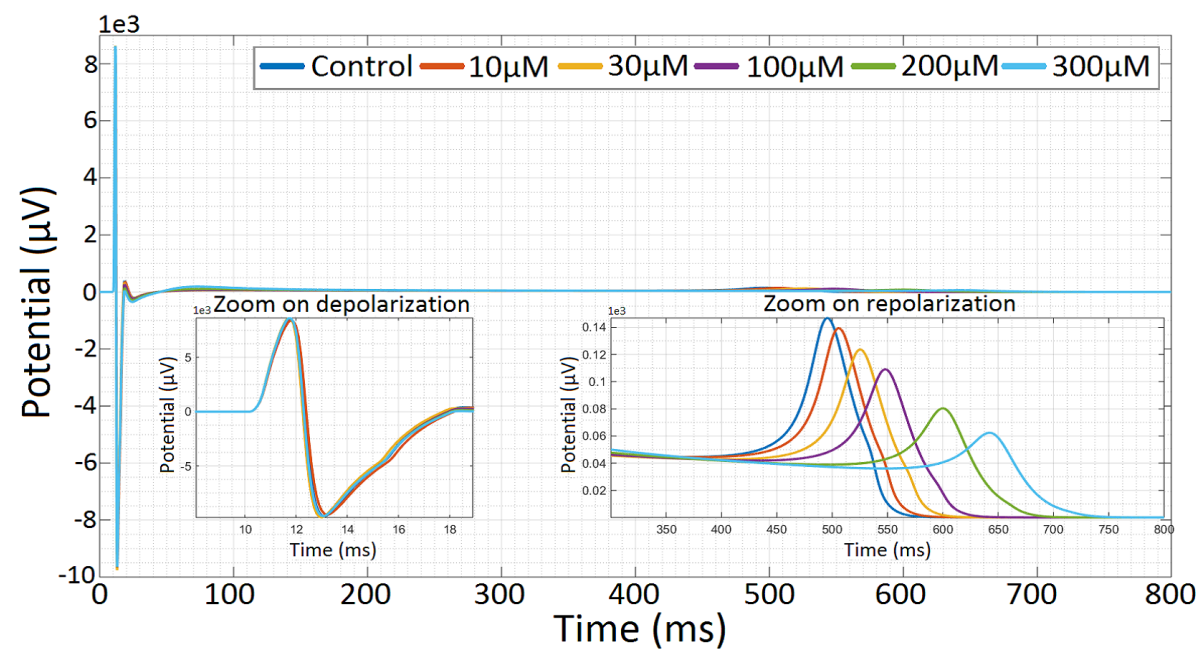

Figure S4: Effects of moxifloxacin on in silico FPs at the end of the identification process using MV model.

\section{S6 Identification results with the Paci model}

In Section III.C of the article, the conductances were estimated for the MV model. We show here that it can also be performed for the Paci model. The identification is restricted here to the single potassium channel. The result is presented in Figure S5 for moxifloxacin. As with the MV model, the agreement with the theoretical curve is very satisfactory. 


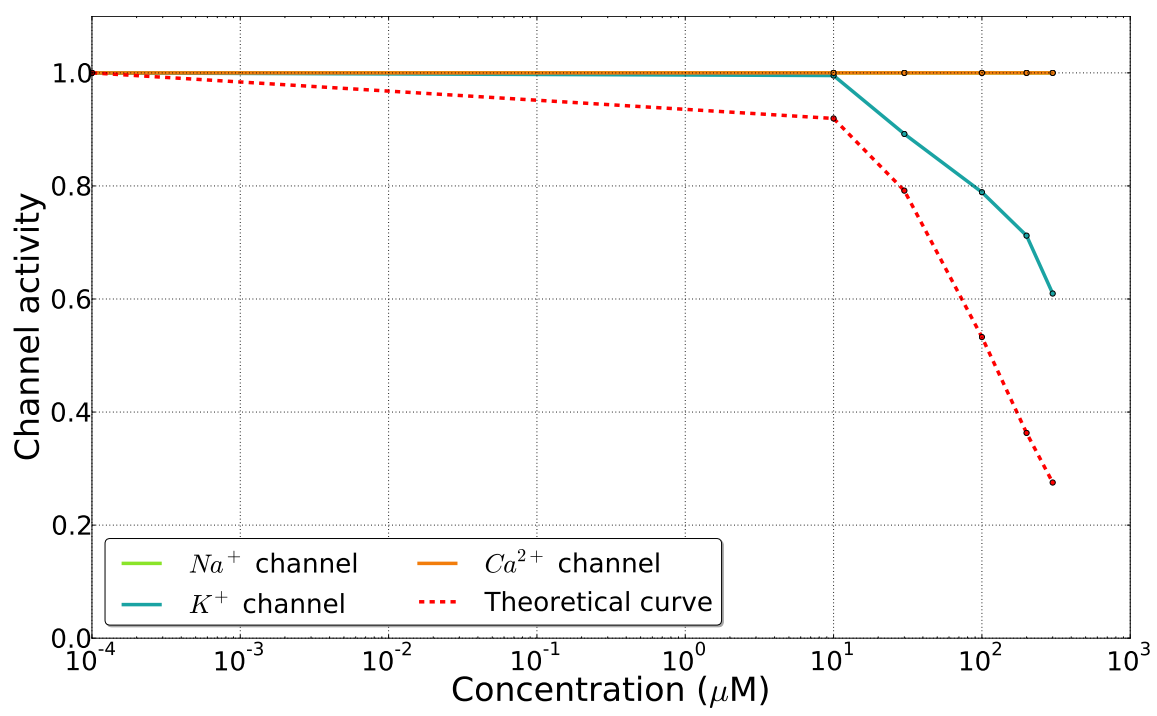

Figure S5: Identification of the moxifloxacin effects on the potassium channel activity using the Paci model.

\section{S7 Computational time}

All the simulations were run on a HP Z820, with 12 processors Intel ${ }^{\circledR}$ Xeon ${ }^{\circledR}$ CPU E5-2640 $0 @ 2.50 \mathrm{GHz}$, and the operating system Linux Ubuntu 12.04.4 LTS 64-bit. The computational time for the forward problems was about 8 minutes with the MV model and 50 minutes with the Paci model. The computational time for the inverse problems was about 20 hours to identify 2 parameters in the MV model, and 17 hours to identify 1 parameter in the Paci model. For the inverse problems, the CMA-ES algorithm was run in parallel on 10 processors. 